\title{
Estrategia Integral de Evaluación para el Mejoramiento del Aprendizaje de la Matemática en Estudiantes de la UNJBG de Tacna en el Año 2009
}

\author{
Integral Strategy of Evaluation for the Improvement of \\ Mathematics Learning of Students at UNJBG in Tacna in 2009
}

' Dionicio Milton Chávez Muñoz, ² Julia Marina Mendoza Gómes

RESUMEN:

La presente investigación tiene por objetivo, determinar en qué medida la aplicación de la estrategia integral de evaluación para el mejoramiento del aprendizaje de la matemática, eleva el nivel de aprendizaje de los estudiantes de ingeniería de la UN/JBG de Tacna en el año 2009; se trabajó con tres grupos de veinte estudiantes cada uno, de tres Escuelas Académico Profesionales de Ingenieria de la UN/JBG de Tacna en el año 2009: usando un diseño cuasi-experimental y la estrategia mencionada, especialmente diseñada para esta investigación, con aplicación reiterada de Pre-Test y Post-Test; sẻ arribó a las siguientes conclusiones: El nivel de aprendizaje de los estudiantes en el ciclo estándar de la estrategia es hajo. Se logró diseñar la estrategia integral de mejoramiento contimuo para el aprendizaje de la matemática y se aplicó al grupo experimental. El nivel de aprendizaje de los estudiantes en el ciclo de mejora contimua de la estrategia, se incrementa obteniendo una media de 9.57 en los promedios finales de evaluación: la diferencia entre los resultados antes y después de la aplicación de la variable independiente es estadísticamente significativa con $\alpha=0.05$.

Palabras clave: evaluación, estrategia integral, mejoramiento contimuo.

\section{ABSTRACT:}

This research aims to determine to what exlent the implementation of the integral evaluation strategy for improving the learning of mathematics, raise the level of learning in engineering students from the UN/JBG in Tacna in 2009: it was worked with three groups of twenty students each. three of the Academic Professional Schools of Engineering at UN/JBG in Tacna in 2009. using a quasi-experimental design and the mentioned above strategy, specially designed for this study, with repeated application of a Pre-Test and Post-Test. The following conclusions were readied: The level of student learning in the standard cycle of the strategy is low; was possible to design a integral strategy of continuous improvement to mathematics learning and was applied to the experimental group. The level of student learning in the cycle of continuous improvement strategy, was increased obtaining 9.57 in the average final evaluation, the difference between the results before and after application of the independent variable is statistically significant at $\alpha=0.05$.

Keywords: evaluation, integral strategy, contimous improvement

\footnotetext{
'Doctor en Fducación con mención en Gestión Educativa, Magister en Docencia Universitaria, Licenciado en Matemáticas.

Facultad de Ciencias. Universidad Nacional Jorge Basadre Grohmann

${ }^{2}$ Magister en Docencia Universitaria, Licenciado en Matemáticas. Facultad de Ciencias. Universidad Nacional Jorge Basadre Grohmann
} 


\section{INTRODUCCIÓN}

En la actualidad los procesos de globalización e internacionalización están exigiendo a las instituciones públicas a reorientar los planes estratégicos y servicios que brindan. Los escenarios internacionales, nacionales, regionales y locales presentan a las universidades retos cada vez más exigentes como: apoyar a que sus paises estén con capacidad científica y tecnológica que soporten la introducción de una economia mundial globalizada; $y$, crear cuadros de profesionales y técnicos que la renovación de las estructuras de producción y de servicios le exijan. Las universidades están iniciando un proceso de acreditación por instituciones nacionales e internacionales, para la evaluación de la calidad en la educación superior en aspectos sociales, cognitivos, habilidades y competencias internas y externas para la formación integral del egresado, con un perfil de competitividad internacional y con sentido humanista. Con la presente investigación se quiere contribuir con la formación intural de los profesionales que van a desempeñarse en el mercado laboral; la estrategia integral de evaluación para el mejoramiento del aprendizaje de la matemática de los estudiantes, tiene como una de sus características, la de ir monitoreando el proceso de enseñanza-aprendizaje, desarrollado por docentes y estudiantes en actividades de clase y extraclase, y está orientada a rescatar y valorar todos los aspectos del desempeño académico-formativo del estudiante.

\section{MATERIALESY MÉTODOS}

\section{Métodos teóricos y de recopilación de datos.}

Uso del método histórico para la sistematización teórica del objeto y campo de investigación para la fundamentación, diseño y elaboración de la estrategia integral que se propone. Uso de otros métodos en la fundamentación y construcción de la estrategia integral: Método histórico-lógico para la evolución histórica del objeto de investigación: el proceso de evaluación de la matemática en las Facultades de Ingenieria elegidas de la UNJBG de Tacna: Método dialéctico-histórico para el estudio del campo de investigación y construcción de la estrategia; Método sintético-estructural y de análisissintesis para diseñar las partes que conforman la estrategia integral y los planes de mejora continua que conforman la estrategia.

\section{Técnicas de recopilación de información.}

La recopilación de la información exigió el uso de las técnicas: Evaluación, Encueta y observación.

\section{Métodos y téenicas de análisis de la información.}

Se necesitó hizo uso de: Análisis documental: El método analítico-sintético: Confrontación de hallazgos con el marco teórico; Técnicas estadísticas con ayuda del SPSS 15 y EXCEL.

\section{Los Instrumentos de recopilación de la in formación.}

Para la recopilación de la información se hicieron necesarios los siguientes instrumentos: El cuaderno de campo: los cuestionarios; registros de observación; registros generales de evaluación y calificacion de exámenes parciales, prácticas calificadas, trabajos encargados, trabajo realizado en aula; presencia en clase, actitudes frente al clesarrollo de la asignatura, etc.

\section{RESULTADOSY DISCUSIONES}

\section{Construcción de la Estrategia.}

La estrategia está plasmada en el siguiente diagrama que se ha denominado: Dinámica para la aplicación de la

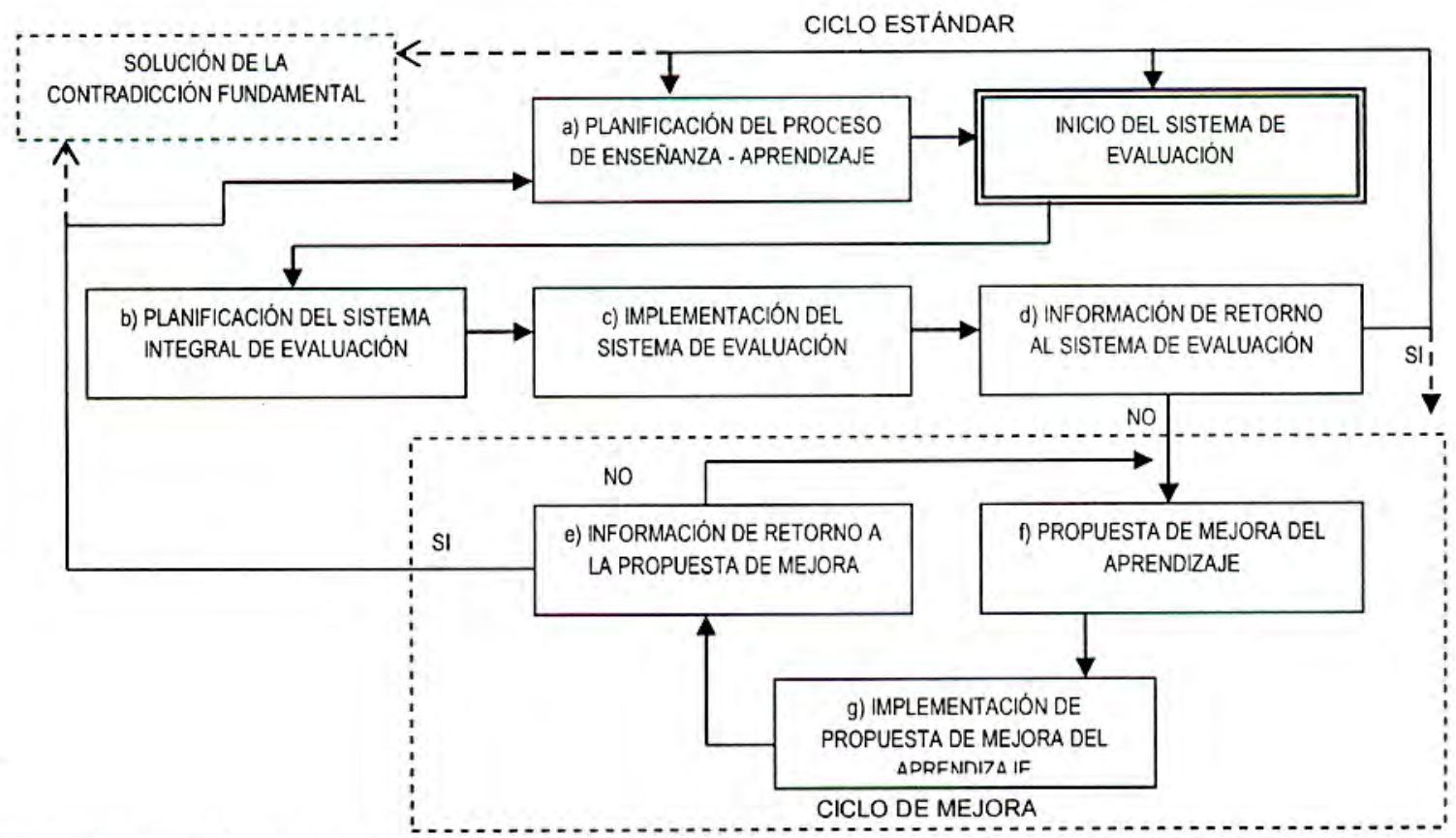

Figura 1. Estrategia Integral de Evaluación para el Mejoramiento del Aprendizaje de la Matemática Fuente: Diseño elaborado por los autores 
estrategia integral de evaluación para el mejoramiento del aprendizaje de la matemática.

\section{Etapas de la estrategia:}

\section{Primera etapa. Ciclo estándar de evaluación.}

Este es el ciclo estándar de la estrategia; consta de cuatro sub-etapas que son:
a) Planificación del proceso de enseñanza-aprendizaje.
b) Planificación del sistema Integral de evaluación.
c) Implementación del sistema integral de evaluación.
d) Información de retorno al sistema de evaluación.

\section{Segunda etapa. Ciclo de mejora del aprendizaje.}

Este es el ciclo de mejora, que se propone para los estudiantes que no han alcanzado los objetivos planificados. Se genera como parte de los informes que se alcanzan a los estudiantes, donde se plantean los planes de mejora según los logros, limitaciones o errores que hayan tenido los estudiantes en el ciclo estándar. -Este ciclo es de libre participación; tiene tres sub-etapas que son:

a) Propuesta de mejora del aprendizaje.

b) Implementación de la propuesta de mejora del aprendizaje.

c) Información de retorno a la propuesta de mejora.

\section{Eficacia de la estrategia.}

Aquí se presentan los resultados obtenidos de la aplicación de la encuesta a los estudiantes del grupo de trabajo. que tuvo el objetivo de determinar tanto la eficiencia cono los resultados de la aplicación de Estrategia Integral de Evaluación para el Mejoramiento del Aprendizaje de la Matemática en lo que corresponde a su fornación académica y humanística. Este indicador de la estrategia integral es cumplido mediante un elevado logro de los siguientes sub-indicadores: la planificación, la aceptabilidad, la flexibilidad, el aspecto integral, el uso óptimo de recursos y el cumplimiento del programa diseñado para aplicar la estrategia.

\section{a) Respecto a la planificación de la estrategia.}

VerTabla 01.

Interpretación. El $85 \%$ dice que tanto la organización como la planificación han sido muy buenas $(50 \%)$ o excelente $(35 \%)$.

Análisis. Los resultados permiten afirmar que la estrategia tiene una adecuada planificación.

\section{b) Respecto a la aceptabilidad de la estrategia.}

\section{Ver Tabla 02.}

Interpretación. El 75\% dice haber estado con buena o muy buena motivación, el $70 \%$ dice haber tenido una buena o regular participación, el $95 \%$ dice haber tenido una buena o muy buena asimilación de contenidos, el $70 \%$ dice haber tenido una buena o muy buena dedicación de tiempo al curso, el $75 \%$ dice haber tenido una muy buena o excelente asistencia a clase.

Análisis. Los resultados permiten afirmar que la estrategia tiene una buena aceptabilidad.

\section{c) Respecto a la flexibilidad de la estrategia.}

Ver Tabla 03.

Interpretación. Los estudiantes tienen opinión muy

Tabla 01. Distribución de estudiantes según su opinión respecto a la planificación.

\begin{tabular}{|c|c|c|c|c|c|c|c|c|c|c|c|c|}
\hline \multirow{3}{*}{$\begin{array}{c}\text { CRITERIO } \\
\text { EVALUADO }\end{array}$} & \multicolumn{10}{|c|}{ OPINIÓN DEL ESTUDIANTE } & \multirow{2}{*}{\multicolumn{2}{|c|}{ Total }} \\
\hline & \multicolumn{2}{|c|}{ Excelente } & \multicolumn{2}{|c|}{ Muy bueno } & \multicolumn{2}{|c|}{ Bueno } & \multicolumn{2}{|c|}{ Regular } & \multicolumn{2}{|c|}{ Malo } & & \\
\hline & $\mathrm{n}$ & $\%$ & $\mathrm{n}$ & $\%$ & $\mathrm{n}$ & $\%$ & $\mathrm{n}$ & $\%$ & $\mathrm{n}$ & $\%$ & $\mathrm{~N}$ & $\%$ \\
\hline La organización del curso & 7 & 35 & 10 & 50 & 3 & 15 & 0 & 0 & 0 & 0 & 20 & 100 \\
\hline La planificación de la evaluación & 7 & 35 & 10 & 50 & 3 & 15 & 0 & 0 & 0 & 0 & 20 & 100 \\
\hline
\end{tabular}

Fuente: Cuestionario aplicado a estudiantes del grupo experimental

Tabla 02. Distribución de estudiantes según su opinión respecto a la aceptabilidad

\begin{tabular}{|c|c|c|c|c|c|c|c|c|c|c|c|c|}
\hline \multirow{3}{*}{$\begin{array}{c}\text { CRITERIO } \\
\text { EVALUADO }\end{array}$} & \multicolumn{10}{|c|}{ OPINIÓN DEL ESTUDIANTE } & \multirow{2}{*}{\multicolumn{2}{|c|}{ Total }} \\
\hline & \multicolumn{2}{|c|}{ Excelente } & \multicolumn{2}{|c|}{ Muy bueno } & \multicolumn{2}{|c|}{ Bueno } & \multicolumn{2}{|c|}{ Regular } & \multicolumn{2}{|c|}{ Malo } & & \\
\hline & $\mathrm{n}$ & $\%$ & $\mathrm{n}$ & $\%$ & $\mathrm{n}$ & $\%$ & $\mathrm{n}$ & $\%$ & $\mathrm{n}$ & $\%$ & $\mathrm{~N}$ & $\%$ \\
\hline Mi motivación ha sido & 0 & 0 & 10 & 50 & 5 & 25 & 5 & 25 & 0 & 0 & 20 & 100 \\
\hline Mi participación ha sido & 0 & 0 & 4 & 20 & 7 & 35 & 9 & 45 & 0 & 0 & 20 & 100 \\
\hline Mi asimilación de contenidos ha sido & 0 & 0 & 10 & 50 & 9 & 45 & 1 & 5 & 0 & 0 & 20 & 100 \\
\hline Mi tiempo dedicado al curso ha sido & 0 & 0 & 5 & 25 & 9 & 45 & 6 & 30 & 0 & 0 & 20 & 100 \\
\hline Mi asistencia a clase ha sido & 9 & 45 & 6 & 30 & 4 & 20 & 1 & 5 & 0 & 0 & 20 & 100 \\
\hline
\end{tabular}

Fuente: Cuestionario aplicado a estudiantes del grupo experimental 
son las acertadas.

- Cuando un estudiante tiene la oportunidad de experimentar el ciclo de mejora de la estrategia y retorna al ciclo estándar, sus limitaciones han quedado superadas.

- Los temas abordados y las estrategias metodológicas que se trabajan en el plan de mejora, son los necesarios para superar el limitado logro de objetivos.

- El aspecto actitudinal mejora sustancialmente, dado que el ingreso al ciclo de mejora es voluntario, el estudiante tiene mayor libertad y seguridad en su actuación con un mayor interés por aprender.

- Respecto a la práctica de valores, se rescatan los de responsabilidad, laboriosidad, respeto mutuo, colaboración y libertad que contribuyen a un mejor trabajo en el grupo.

- El ciclo estándar de evaluación y el ciclo de mejora se complementan y en conjunto hacen que los objetivos de la investigación que se sustentan en mejorar el aprendizaje y elevar los rendimientos se cumplan.

- Los aprendizajes tienen duración más prolongada, esto se corrobora con la aplicación de pruebas luego de un ciclo de terminada la experiencia.

\section{CONCLUSIONES}

1. El nivel de aprendizaje de los estudiantes en el ciclo estándar de la estrategia, es bajo con una media de 8.19; para los promedios finales de evaluación.

2. Se logra elaborar la estrategia integral de evaluación para el mejoramiento del aprendizaje de la matemática y se aplicó a tres grupos durante el desarrollo de la investigación.

3. El nivel de aprendizaje de los estudiantes en el ciclo de mejora de la estrategia se incrementa obteniendo una media de 9.57; para los promedios finales de evaluación.

4. Los resultados obtenidos en el ciclo estándar son menores que los resultados obtenidos en el ciclo de mejora, siendo está una diferencia estadísticamente significativa con un $\alpha=0.05$; para los promedios finales de evaluación.

5. Los resultados obtenidos en el ciclo estándar son menores que los resultados obtenidos en el ciclo de mejora, siendo está una diferencia significativa con un $\alpha=0.05$; para los exámenes parciales; para las prácticas calificadas; para los trabajos encargados; y para el trabajo realizado en aula.

\section{REFERENCIAS BIBLIOGRÁFICAS}

Ahumada Acevedo, Pedro. 2001. La evaluación en una concepción del aprendizaje significativo. Ediciones Universitarias de Valparaiso. Chile. Pág. 18.

Alfaro, M. E. 1990. "Aspectos prácticos del proceso de programación y evaluación". Documentación Social. $\mathrm{N}^{\circ}$ 81. Madrid. Pág. 70.

Alvaréz de Zayas, Carlos. 1992. La Escuela en la vida. Editorial Félix Varela. Colección Educación y desarroilo. La Habana.

Brown, Rally y Glasner, Angela. 2003. Evaluar en la universidad. Ediciones NANCEA S.A. Madrid-España, Pág. 180.

Casanova, María Antonia. 2002. Manual de Evaluación Educativa. 8va. Edición. Editorial La Muralla. Madrid.

Camperos, M., 1984. La evaluación formativa del aprendizaje. Mimeo. Caracas-Venezuela.

Cano de Canales, Yolanda. 1991. Los Instrumentos como Ayuda del Aprendizaje. Editorial Supergráfica. Linıa Perú.

\section{Correspondencia:}

Dionicio Milton Chávez Muñoz

Ciudad Universitaria Fundo "Los Granados"

Av. Miraflores s/n. Tacna. Perú

miltondioni@hotmail.com 